\title{
Viewpoints
}

\section{The Tubular Striatum}

\author{
${ }^{(-D}$ Daniel W. Wesson \\ Department of Pharmacology \& Therapeutics, University of Florida, College of Medicine, Gainesville, Florida 32610
}

In the mid-19th century, a misconception was born, which understandably persists in the minds of many neuroscientists today. The eminent scientist Albert von Kölliker named a tubular-shaped piece of tissue found in the brains of all mammals studied to date, the tuberculum olfactorium - or what is commonly known as the olfactory tubercle (OT). In doing this, Kölliker ascribed "olfactory" functions and an "olfactory" purpose to the OT. The OT has since been classified as one of several olfactory cortices. However, further investigations of OT functions, especially over the last decade, have provided evidence for roles of the OT beyond olfaction, including in learning, motivated behaviors, and even seeking of psychoactive drugs. Indeed, research to date suggests caution in assigning the OT with a purely olfactory role. Here, I build on previous research to synthesize a model wherein the OT, which may be more appropriately termed the "tubular striatum" (TuS), is a neural system in which sensory information derived from an organism's experiences is integrated with information about its motivational states to guide affective and behavioral responses.

Historical conceptualization of the olfactory tubercle

Neurobiology, like all branches of science, has developed its fair share of misconceptions. These misconceptions may have origins as far back as the late 18th century when Franz Joseph Gall sparked assumptions that core aspects of brain function, including affect and cognition, are localized to certain brain areas (Simpson, 2005). More specific pursuits to assign functions were made as histologic staining techniques allowed neuroanatomical pioneers to identify the complex anatomic organization of the brain. For instance, the nucleus accumbens was once designated a component of what was area parolfactoria, and specifically was termed the nucleus parolfactorious lateralis (note the olfactory inclusion in its name) (Johnston, 1913) simply based on its physical location, thereby assigning it an olfactory function (Johnston, 1923). Later, the nucleus accumbens was also called the "olfacto-striatum" (Herrick, 1926), despite no evidence for input from the olfactory bulb. This contextualization of the nucleus accumbens has certainly been corrected, especially over the past decades, with mountains of anatomic, behavioral, and physiological evidence indicating the nucleus accumbens is an integral component of the basal ganglia (for review, see Carelli, 2002; Kalivas and Volkow, 2005; Nicola, 2007; Floresco, 2015).

In the mid-19th century, a similar misconception, which persists in many minds today, occurred when the eminent and pioneering scientist Albert von Kölliker named a tubular-shaped

\footnotetext{
Received May 8, 2020; revised Aug. 10, 2020; accepted Aug. 10, 2020.

The author declares no competing financial interests.

This work was supported by National Institutes of Health Grants R01DC014443, R01DC016519, R01DA049545, and R01DA049449. I thank members of my laboratory who have contributed research on this subject, especially Drs. Marie Gadziola, Kaitlin Carlson, Luke Stetzik, Katherine Wright, Estelle In 't Zandt, and Hillary Cansler; and the wonderful collaborators and mentors whom I have shared discovery with on this particular topic, including Drs. Minghong Ma, Don Wilson, Nathalie Mandairon, Mariella De Biasi, Fuqiang Xu, Lori Knackstedt, Marc Fuccillo, as well as members of their laboratories.

Correspondence should be addressed to Daniel W. Wesson at danielwesson@ufl.edu.

https://doi.org/10.1523/JNEUROSCI.1109-20.2020

Copyright $\odot 2020$ the authors
}

piece of tissue on the most ventral part of the brain, the tuberculum olfactorium - or what is now commonly known as the olfactory tubercle (abbreviated as either Tu or OT) (Kölliker, 1896). While Kölliker was a leader in some aspects of physiology, in addition to his anatomic contributions, this naming of the OT as an olfactory structure was not based on physiological evidence or function. It was based on the OT's tubular shape and its proximity to the olfactory bulb (Kölliker, 1896). Nevertheless, in doing so, Kölliker assigned the OT "olfactory" functions, and an "olfactory" purpose, which has galvanized how brain researchers have conceptualized the OT for more than a century.

It is not the intention of this Viewpoints article to say the OT is void of olfactory roles. As summarized later, the OT is indeed both richly interconnected with other olfactory structures, and partakes in processing odor information. Thus, it was not a tremendous disservice for Kölliker to have ascribed olfactory functions to the OT. Instead, the goal of this Viewpoints article is to summarize recent advances indicating that the view of the OT as solely an "olfactory" structure is too narrow and restrictive to guide research in fully understanding the contributions of this brain region to the neurobiology of behaviors.

\section{Early anatomy confirmed the OT is interconnected within} the olfactory system

The early anatomic work that included the OT in its sights is rich and spans a wide array of vertebrate animals. In most animals, the OT can be observed as the round (i.e., "tubular") bulge on the most ventral aspect of the basal forebrain. In rodents and birds, for instance, the OT is visually apparent when inspecting the inferior view of the brain (Fig. 1), being a pronounced bulge between the lateral olfactory tract and the optic chiasm. In humans, the OT may be called the anterior perforate space or the anterior perforated substance, which, since the time of Ramon y Cajal (1904), has been considered a conserved brain region from lower vertebrates to humans. In humans and many nonhuman primates, the OT is in a similar portion of the basal 
a

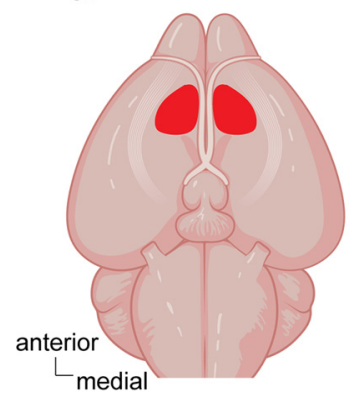

b

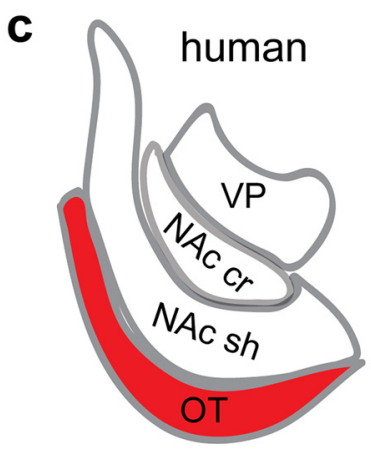

mouse

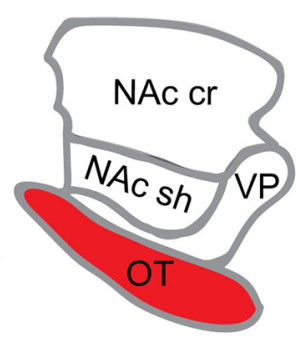

Figure 1. Localization of the OT in mice and humans. Approximate areas of the OT in the mouse brain from both the inferior $(\boldsymbol{a})$ and coronal $(\boldsymbol{b})$ views. $\boldsymbol{c}$, Illustration of the relative location of the $0 \mathrm{~T}$ in humans and mice in respect to the neighboring nucleus accumbens shell (NAc sh) and core (NAc cr) and the ventral pallidum (VP).

forebrain, yet often not visible on the external inferior surface of the brain.

The OT is not a homogeneous structure but instead has a highly elaborate cytoarchitecture. This is related to its composition of compartments (cortical, striatal). These are well defined in larger mammals in which the cortical compartment can be observed in the more lateral aspect of the OT and the "striatumlike" compartment in the medial aspect (Meyer and Wahle, 1986). In the cortical compartment, the cell layers undulate, giving rise to so-called "caps" of cells (Meyer and Wahle, 1986). Adding to this heterogeneous composition is the presence of dense clusters of granule cells termed the islands of Calleja (Ganser, 1882; Millhouse and Heimer, 1984). Although the islands of Calleja are observed in animals ranging from rodents to primates, it is notable that the discrete borders and presence of the OT's compartments are not highly evident in small rodents, like mice.

The OT is composed of multiple cell types (Millhouse and Heimer, 1984; Millhouse, 1987), each with distinct electrophysiological properties (Chiang and Strowbridge, 2007). As will become more apparent below, the vast majority of OT neurons are medium spiny neurons (also known as spiny projection neurons). This cellular composition differs from most olfactory areas, in which the principal neurons are glutamatergic pyramidal cells.

Kölliker's presumption that the OT is an "olfactory" structure manifested as true in the sense that, when anatomic tracing methods arose, the findings supported the dense connectivity of the OT with the olfactory system (Fig. 2a). The OT receives massive input from the olfactory bulb: directly from its output neurons, called mitral and tufted cells (Gurdjian, 1925; L. E. White, 1965; Scott et al., 1980). The OT also receives disynaptic input from the olfactory bulb by way of the piriform cortex (Schwob and Price, 1984a; Carriero et al., 2009; K. A. White et al., 2019), which like the OT, receives direct input from olfactory bulb output neurons. Nearly the entirety of the OT, which spans quite a large space along the ventral surface of the brain (Fig. 1a), receives input from an olfactory brain region (L. E. White, 1965; Heimer, 1968; Luskin and Price, 1983a,b; Schwob and Price, 1984a,b; Johnson et al., 2000; Sosulski et al., 2011; Zhang et al., 2017b). The OT's receipt of monosynaptic input from the OB, together with its largely laminar architecture (three layers), resulted in the OT being classified as one of several olfactory cortices (Pigache, 1970).

\section{Functional evidence for a role of the $\mathrm{OT}$ in olfaction in rodents and humans}

The OT's functional connections with olfactory structures were largely ignored and are still underappreciated compared with the wealth of interest invested into the "primary" olfactory cortex (the piriform) and the olfactory bulb. John Scott and his group were among the first to investigate the functional sources of olfactory input to the OT. They recorded from olfactory bulb mitral and tufted cells, looking for antidromic activation of these cells during stimulation of the OT in anesthetized rats (Scott, 1981). The authors uncovered evidence for functional input from these olfactory bulb cells and, through subsequent horseradish peroxidase injections in the OT, concluded that the cells innervating the OT are mostly tufted cells. Further, the authors reported that antidromic activation of the lateral aspect of the OT, which most closely neighbors the lateral olfactory tract, tended to result in greatest antidromic responses, thus suggesting spatial organization in the functional input from the olfactory bulb. Notably, it is thought, based on tracing work from other groups, that the OT does not innervate the piriform cortex (Zhang et al., 2017b) and thus this activation does not likely result from disynaptic antidromic influences. This was complemented by subsequent work that showed synaptic potentials in the OT after stimulation of the lateral olfactory tract, which is composed of fasciculated olfactory bulb mitral and tufted cells (McNamara et al., 2004). Given these features, it is not surprising that OT local field potential activity is coupled with both nasal respiration and respiratory theta rhythm in the olfactory bulb (Carlson et al., 2014).

Additional research has uncovered functional input to the OT from the piriform cortex. The first paper to do so found, through field potential and voltage sensitive dye imaging of the $\mathrm{OT}$ in an in vitro isolated guinea pig brain, that stimulation of the piriform cortex elicits evoked potentials in the OT, which are nearly abolished after lesion between the piriform and the OT was used to sever association fibers arising from the piriform cortex (Carriero et al., 2009). Follow-up work involving our group with collaborators recently explored piriform association fiber influences on the OT in awake mice. This revealed that evoked stimulation of piriform terminals in the OT influences OT unit firing and that piriform association fibers synapse on OT medium spiny neurons expressing dopamine receptors D1 and $\mathrm{D} 2$, the most common cell types in the OT (K. A. White et al., 2019).

Several groups over the past decade have begun to characterize the representation of odors in the OT without regard to whether the signals originate from the $\mathrm{OB}$, the piriform cortex, or (more likely) a combination of the two sources. Odor-evoked activity arrives in the OT on average $\sim 125 \mathrm{~ms}$ after the onset of inhalation (Payton et al., 2012) (Fig. 2c). Individual OT neurons display broader tuning for some odors than for other odors (Fig. $2 b$ ), but it does not appear OT neurons as a population are 

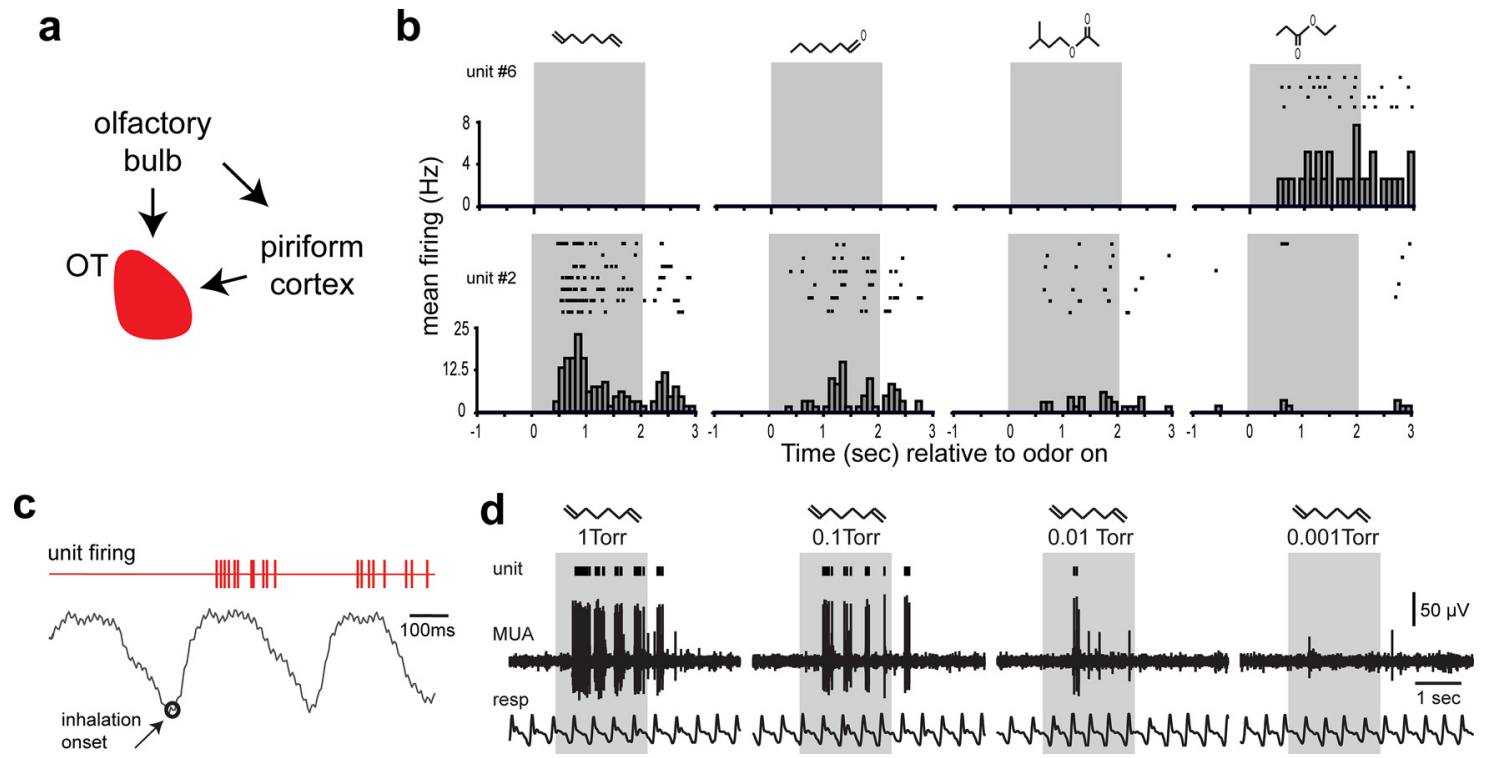

Figure 2. Odor input into the $0 \mathrm{~T}$ and the representation of odors by $0 \mathrm{~T}$ neurons. $\boldsymbol{a}$, Schematic of the major sources of odor information into the $0 \mathrm{~T}$, including from the olfactory bulb and piriform cortex, which receives direct input from the olfactory bulb to extend disynaptic input to the OT. Data from Gurdjian (1925), L. E. White (1965), Scott et al. (1980), and Schwob and Price (1984b). $\boldsymbol{b}$, Example of odor-evoked activity in two 0T single units from two separate urethane-anesthetized mice. Data are single-unit raster plots and peristimulus time histograms across multiple presentations with four different odorants (from left to right: 1,7-octadiene, ethyl propionate, heptanal, and isoamyl acetate). The top unit displays narrow tuning, responding to only one of the odorants, whereas the bottom unit responds to several odorants. Data adapted from Wesson and Wilson (2010). c, Example single-unit raster of spiking events (red) during odor presentation in relation to respiratory cycles, indicating the short latency until spiking from this unit relative to the onset of inhalation (open circle). Data adapted from Payton et al. (2012). $\boldsymbol{d}$, Example multiunit $0 \mathrm{~T}$ trace (MUA) and single-unit raster plot (unit) along with respiration (resp) from a single recording in a urethane-anesthetized mouse showing reduction in spiking with decreased intensities of a single odor (1,7-octadiene). Whereas the 1 torr intensity odor elicited robust spiking during most inhalation events, the single unit in this example did not spike to the lowest intensity. Data adapted from Xia et al. (2015).

specialized to represent any given type of odor (e.g., social, food) because OT neurons respond to all odor types and odorants studied to date (Wesson and Wilson, 2010; Payton et al., 2012). OT neurons represent odors based on their intensity (Fig. 2d) and can display adaptation to odors over prolonged exposure, similar to the piriform cortex (Xia et al., 2015). Stimulus selective, concentration variant, and/or adapting responses to prolonged stimulus exposure are several of the hallmark features for sensory coding observed throughout primary and secondary sensory processing nodes. Notably, while the above studies were all in mice or rats, early work in nonhuman primates suggested the presence of odor-evoked activity in the OT (Williams et al., 1993). Thus, in the context of odors, the OT acts like an olfactory structure in its odor responding.

Interestingly, work from both rats and humans has begun to uncover insights into how odor representations in the OT may be shaped by behavioral states (Carlson et al., 2014), and even specific cognitive states, such as selective attention (Zelano et al., 2005; Carlson et al., 2018). Because cognitive modulation of sensory processing is observed throughout sensory cortices (Desimone and Duncan, 1995; Lakatos et al., 2007; Fontanini and Katz, 2008), this work provides additional evidence that OT has properties comparable to those seen throughout sensory systems.

\section{Counterpoint: the ventral striatopallidum}

The above evidence leaves no doubt that the OT partakes in representing odors and in the state-dependent modulation of those representations in manners that would enhance perception based on behavioral and cognitive demand. However, in the mid-tolate 20th century, the anatomist Lennart Heimer contested the notion that the OT should be grouped with the piriform cortex as a component of the olfactory system. Heimer's work on this topic, which will be briefly reviewed here, led to a different perspective on the OT, which has since been investigated in the context of motivated behavior.

Heimer and colleagues put forth several lines of reasoning explaining why the OT should be considered part of the ventral striatum (Heimer et al., 1982; Switzer et al., 1982; Alheid and Heimer, 1988; De Olmos and Heimer, 1999). Heimer performed lesion studies that, through investigation of degeneration of interconnected structures, indicated that the OT was more connected with regions beyond the olfactory system than with the neighboring piriform cortex. Heimer further argued that the OT was truly "striatal" in nature because, like the striatum, it was composed largely of medium-sized (spiny) neurons that, in some areas of the OT, formed a patchy organization. He also noted that, similar to the rest of the striatum, the OT is rich in acetylcholinesterase. Indeed, inspection of a brain atlas, including acetylcholinesterase staining, quite remarkably allows clear identification of the OT at the very ventral part of the brain, beneath the nucleus accumbens, and disambiguates the OT from its medially and laterally neighboring structures (i.e., the ventral diagonal band and the piriform cortex) (Paxinos and Franklin, 2000). Additionally, Heimer noted afferent and efferent projections to and from the OT are analogous to those of other striatal structures, including input from the globus pallidus and dense innervation by ventral tegmentum dopaminergic neurons in the midbrain. Further, the OT, like the nucleus accumbens, projects to the ventral pallidum and receives massive cortical input, including from the frontal cortex (Vertes, 2004; Hoover and Vertes, 2011; Zhang et al., 2017b), as well as input from the amygdala and ventral subiculum. The most overt distinction between the OT and other striatal structures, such as the nucleus accumbens in terms of connectivity, is that only the OT receives direct synaptic input from the olfactory bulb. 


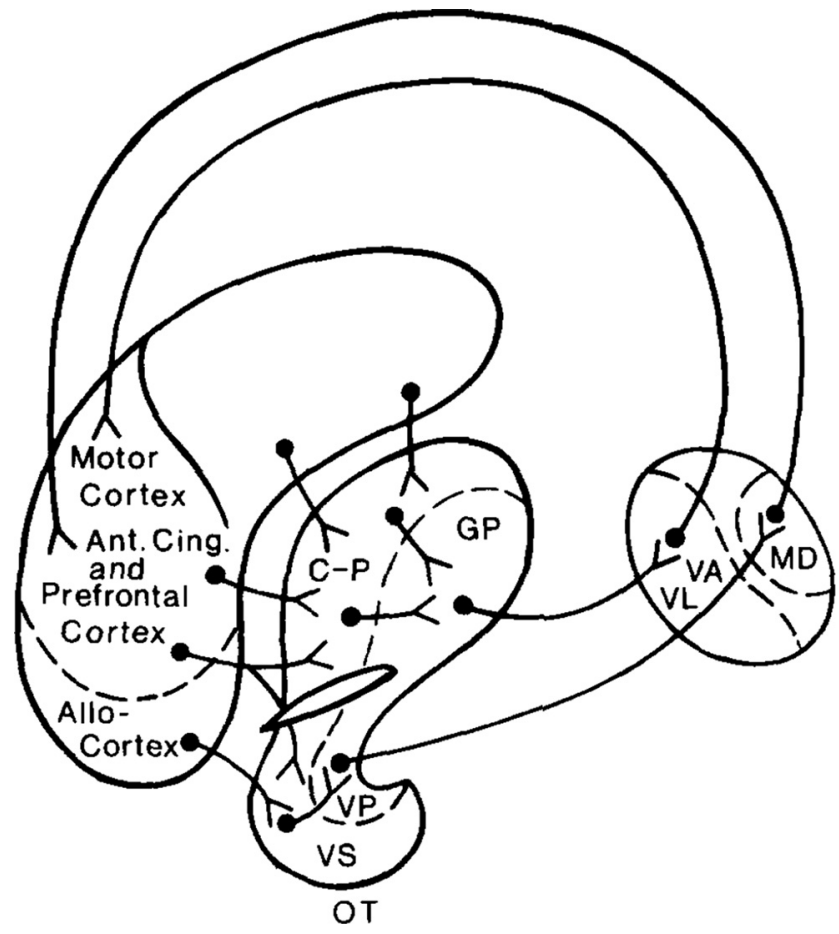

Figure 3. Lennart Heimer's original drawing, illustrating his concept that the ventral striatopallidum is integral with the basal ganglia's striato-pallidopthalamic loop. Reproduced with permission from Heimer et al. (1982). VS, Ventral striatum; VP, ventral pallidum; MD, medio-dorsal thalamus; VA, ventralis anterior complex of the thalamus; VL, ventralis lateralis complex of the thalamus; GP, globus pallidus; $C-P$, caudate putamen. While not indicated in this illustration, Heimer included the nucleus accumbens as part of the VS.

Together, this work led Heimer to propose that the nucleus accumbens and OT form a conglomerate brain system he termed the ventral striatopallidum, which today is more commonly called the ventral striatum (though notably this term is not overtly inclusive of the ventral pallidum). Noting connectivity of the ventral striatopallidum, which contributes to the basal ganglia's striato-pallidothalamic loop (Fig. 3), Heimer posited that the ventral striatopallidum influences behavior, including motor behaviors (Heimer et al., 1982). Further, he predicted that the ventral striatopallidum's subsystems (the nucleus accumbens, ventral pallidum, and OT), while interconnected (for review, see Ikemoto, 2007), play distinct roles in behavior based on their anatomy and neurochemistry (De Olmos and Heimer, 1999). As a side note, Herrick's "olfacto-striatum," which, as mentioned earlier in this paper, was the term he used for the nucleus accumbens, did not encompass the OT (Herrick, 1926).

\section{Evidence supporting involvement of the OT in motivation,} reward, and addiction

What evidence might support Heimer's concept of the OT as a component of the ventral striatum? In 1978, George Koob and colleagues lesioned dopaminergic innervation of both the OT and the nucleus accumbens with 6-hydroxydopamine, and reported that rats with lesions consumed more food and displayed less switching between eating and drinking modes than is typical (Koob et al., 1978). Prado-Alcalá and Wise (1984) allowed rats to self-administer electrical stimulation of the OT and reported that such stimulation was rewarding. Our group followed up on this work many years later by showing that electrical stimulation of the OT in mice is sufficient to influence place preferences (Fitzgerald et al., 2014).
The Prado-Alcalá and Wise (1984) paper noted above also reported that rats would self-administer electrical stimulation to other brain areas, including the nucleus accumbens. What still remained to be determined was whether the OT and nucleus accumbens may differ in how they may distinctly contribute to reinforcement behavior. In 2003, Satoshi Ikemoto performed a careful and elegant experiment to assess whether rats would selfadminister cocaine intracranially into the OT (Ikemoto, 2003). Ikemoto implanted rats with infusion cannulae into the nucleus accumbens core or shell or into discrete areas of the OT, including the medial and lateral aspects. He reported evidence in support of Heimer's hypothesis the ventral striatum subregions may have unique functions by demonstrating that rats would selfadminister cocaine into the OT more often than into the nucleus accumbens (Fig. 4a). Further, he found evidence that specialized regions within the OT support this behavior: rats pressed for cocaine infusions into the medial OT more than into the lateral. This study by (Ikemoto, 2003) is to date the gold-standard work on the reinforcing effects of the OT and one that has been clearly influential in many of the subsequent studies that will be discussed here.

More recent work has shown that OT neural activity represents instrumental actions, as well as rewards themselves. First, and related to the above work by (Ikemoto, 2003), OT activity is modulated around the times animals self-administer cocaine (Striano et al., 2014). Second, work by our group found that OT units in water-motivated mice represent instrumental responding (licking) to obtain a fluid reward (Gadziola and Wesson, 2016) (Fig. 4b,c). The representation occurs before lick onset (Fig. 4c), and this "invigoration" of spiking is dependent on the motivation state of the animal with more spiking occurring when animals are less thirsty. Further, the firing of OT units encodes reward intake (but interestingly not reward omission) (Fig. $4 b$ ), the type of reward taken (at least when comparing natural reinforcers, e.g., saccharin, sucrose, or water), and also the reward magnitude (Gadziola and Wesson, 2016).

Notably, comparable features of neural activity to those reviewed above are observed in the nucleus accumbens (e.g., Carelli et al., 1993; West and Carelli, 2016). How might OT neural activity differ from that in the nucleus accumbens? Recent work from our group sought to define functional differences between these ventral striatum subregions in the encoding of instrumental responding for rewards, as well as rewards themselves (Wright and Wesson, unpublished observations). We performed simultaneous recordings of OT and nucleus accumbens single-unit activity as mice engaged in a sucrose self-administration task, followed by extinction training and cue-primed reinstatement task phases. Importantly, for this to be a fair comparison (since the OT receives massive olfactory input), the cues used were not olfactory, but instead were auditory and visual. As expected, neurons in both regions represented instrumental responding and reward acquisition. However, throughout the task phases, more OT neurons were significantly modulated in their firing compared with those in the nucleus accumbens. Further, compared with the nucleus accumbens, among those modulated neurons, those in the OT displayed greater changes in firing that coincided with both anticipatory behavior and consummatory behavior when reward was available. Throughout extinction training and cue-primed reinstatement (i.e., when reward was not available), OT neurons that were recruited still displayed some greater increases in firing than those in the nucleus accumbens. These data suggest there are differences in how ventral striatum subregions are engaged during motivated behavior (Wright and Wesson, unpublished 


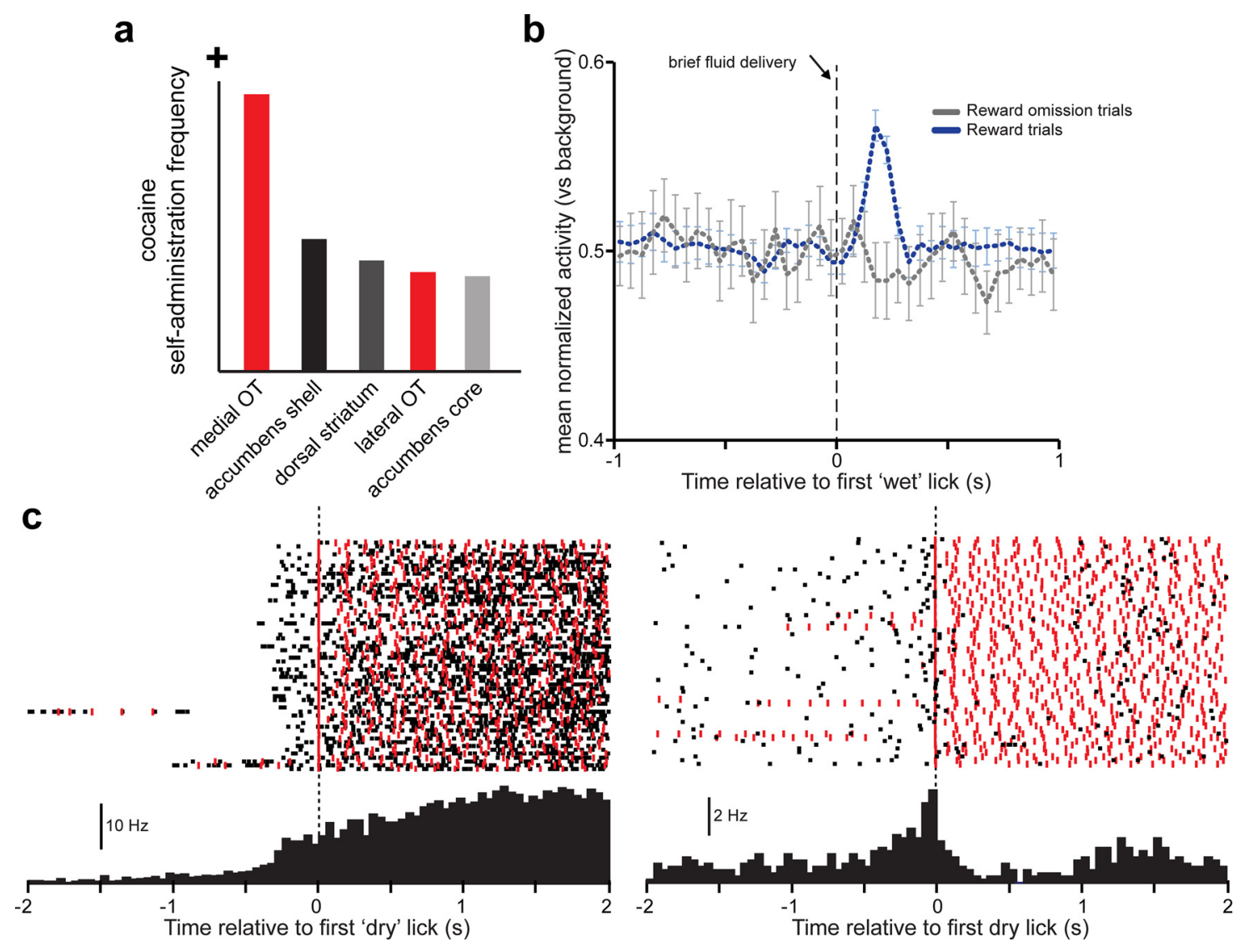

Figure 4. Evidence that the $0 T$ supports the reinforcing properties of psychoactive drugs and encodes rewards. $\boldsymbol{a}$, Summary of results from the work of Ikemoto (2003), indicating the frequency of intracranial cocaine infusions into ventral striatum subregions (red represents $0 \mathrm{~T}$ ) when rats were allowed to self-administer $200 \mathrm{~mm}$ cocaine. Rats more frequently self-administered cocaine into the medial $0 \mathrm{~T}$ than any other brain region tested. $\boldsymbol{b}$, Population-averaged firing of $0 \mathrm{~T}$ single units (normalized) during the engagement of mice in a water-motivated task requiring them to lick in succession to obtain a fluid reward. Receipt of reward on the first lick ("wet" lick) evoked a brief bust of firing in the units; this was not observed on trials in which the reward was withheld (omission). $c$, Example activity of two single units from separate mice (black rasters across trials and peristimulus time histograms averaged across trials) recorded in the same paradigm as in $\boldsymbol{b}$, but relative to the time the animal initiated the successive licks yet received no reinforcer ("dry" lick). Red ticks represent timing of licks. Both example units increased firing before the onset of the instrumental response to obtain the reward; and in the example to the right, the firing was not related to timing of licks. $\boldsymbol{b}, \boldsymbol{c}$, Adapted with permission from Gadziola and Wesson (2016).

observations), and it is likely these differences will stand out even more in the context of reinforcers with strong olfactory components (e.g., food, cigarettes, alcohol).

Evidence supporting the unique capacity of the OT to shape odor information based on associated values

Some studies provide evidence explaining why an "olfactory" structure appears to have a privileged home in the ventral striatum compared with all other areas that receive olfactory-bulb input. For one, our above-mentioned study on electrical stimulation of the OT in mice (Fitzgerald et al., 2014) reported that OT stimulation is not just reinforcing, but also influences place preferences among odors. Several different laboratories each reported in separate studies that lesions or inactivation of the OT and/or the ventral striatal pallidum, reduced preferences mice displayed for urinary odors of opposite sex conspecifics (Agustín-Pavón et al., 2014; DiBenedictis et al., 2014, 2015), yet without disrupting the basic abilities of the mice to detect and differentiate between odors (DiBenedictis et al., 2014). Perhaps tying these results in with those of the Ikemoto (2003) cocaine infusion study, the lesions in one of these studies were selective to the medial part of the OT (DiBenedictis et al., 2015), again highlighting a likely important role for this OT region in motivation. Indeed, a recent study found that optogenetic manipulation of dopaminergic input to the medial OT is highly crucial in shaping odor preferences, an effect prevented by blocking OT dopamine receptors (Zhang et al., 2017a). This is consistent with ex vivo brain-slice recordings that found phasic dopamine robustly facilitates plasticity at synapses between olfactory-bulb afferents and OT medium spiny neurons (Wieland et al., 2015). Notably, midbrain dopamine neurons may also corelease glutamate, and dopamine-neuron glutamatergic synapses are reported in the OT (Mingote et al., 2015).

Associating odors with learned outcomes profoundly shapes how they are represented in the OT. In 2015, our group performed the first recordings of OT single units in behaving mice and discovered that most OT units represent odors that have been conditioned to predict a reward with greater changes in firing rates than those conditioned not to predict reward (Gadziola et al., 2015). Moreover, through reversal learning, we showed that these responses were flexible: the units tracked the associated outcomes by changing their firing rates as the animals learned the new behavioral contingencies. Also, the activity of OT units reflected subsequent odor-guided behaviors, that is, whether the animal made a correct response or not. At about the same time, a similar report came from Murata et al. (2015) who conditioned mice to associate odors with specific outcomes (food reward or shock) and, using c-Fos mapping, found that the medial aspect 
of the OT was most activated by learned-rewarded odors, whereas the lateral aspect of the OT was most activated by shock-associated odors. Both effects, regardless of associated outcome (reward or shock), were driven largely by odor-induced activation of neurons expressing the D1 receptor. The differential representation of perceived odor "value" reported by the above studies is also supported by human fMRI work in which different odors are paired with uniquely valued outcomes (Howard et al., 2016).

Further supporting the hypothesis that the OT is striatal in nature, recent papers have revealed that the OT is distinct from olfactory regions in its ability to display reward-associated odor coding. For instance, our group and that of Millman and Murthy (Gadziola et al., 2020; Millman and Murthy, 2020) have each recently reported that, whereas OT neurons divergently represent odors conditioned to predict reward more than those that do not, neurons in the piriform cortex do not, or do so to a far lesser extent. The Murthy group showed that the learning of odor-reward association by OT units was exceptionally rapid throughout conditioning (Millman and Murthy, 2020). Further, our group used fiber photometry of aggregate calcium signals in either D1- or D2-Cre mice to discover that the representation of odor-reward associations seems to be driven largely by OT D1 neurons (similar conceptually to the findings reported by Murata et al., 2015) and that optogenetic activation of OT D1 neurons promotes the engagement of mice in a reinforcementbased task (Gadziola et al., 2020). Finally, recent work pairing optical stimulation of midbrain dopamine neurons with odors (in awake, but nonbehaving mice) found, excitingly, that the ability of OT units to drive divergent responding to one odor over another may rely solely on dopaminergic input (Oettl et al., 2020).

Thus, the OT represents rewards, and its activity in response to sensory stimuli is shaped by learned outcomes, not unlike effects seen in addiction, in which cues begin to take on meaning of reinforcer availability. The OT also supports cocaine taking. It is worth noting that, despite these features, current models of the brain's "reward system" do not include the OT (O'Connell and Hofmann, 2011; Russo and Nestler, 2013).

\section{Synthesis of a viewpoint}

Bringing together these lines of research, it is apparent that the OT functions neither as a purely "olfactory" structure nor as a purely "striatal" structure. OT neurons encode odors, but this encoding can depend on associative outcomes: a feature similar to that seen in the striatum's nucleus accumbens (Gottfried et al., 2002). But that said, numerous brain systems represent stimuli depending on their associated valences (Anderson et al., 2003; Zald, 2003; Kumar et al., 2012; Chikazoe et al., 2014), so that does not necessary rule out an alternative framework for the OT. The OT supports cocaine-taking, encodes goal-directed behaviors, and even encodes rewards themselves (Gadziola and Wesson, 2016) in striatal-like manners (Carelli et al., 1993; West and Carelli, 2016). Yet this does not indicate the OT is purely striatal in function and thus dedicated to driving actions and evaluating their outcomes.

What then is the function of the OT? Based on the above studies, I propose that the OT is a neural system in which sensory information derived from an organisms' experiences is integrated with information about its motivational states to guide affective and behavioral responses. By this definition, the OT does not need to be packaged as either olfactory or striatal. Sure, the OT is uniquely positioned to link odor information with motivated states and allow animals to act on those stimuli depending on their motivation. But the OT also receives a variety of other types of sensory inputs through either direct or disynaptic pathways (not reviewed here; but see Wesson and Wilson, 2011). In this framework, the OT may evaluate a wealth of sensory inputs based on motivational states, albeit with a likely bias toward evaluating odor input, given the massive input of odor information it receives.

It is tempting to bestow on the OT a different name. Some groups, including ours, have recently referred to the OT as the "olfactory striatum" (K. A. White et al., 2019; Millman and Murthy, 2020). This brings back memories of the nucleus accumbens once being called the "olfacto-striatum" (Herrick, 1926). While this is certainly more appropriate for the OT than the nucleus accumbens, still, olfactory striatum imposes an "olfactory" function on the OT and misleads the field into the same misconception that Kölliker generated with the name OT. The OT deserves a suitable name that does not predict its to-be-determined functions.

It is apparent the OT is in the striatum; there is no need to question the pioneering work of Heimer, which I will add, was recently advanced by an exceptionally careful modern developmental study that indicated that OT neurons have developmental lineages similar to other ventral striatum neurons, and distinct from olfactory neurons (Martin-Lopez et al., 2019). The OT is also notably "tubular" in shape. If an alternative name would be raised going forward, I would propose the "tubular striatum" (TuS). Rebranding the OT as TuS may result in some confusion for the immediate future, yet the long-term benefits of helping the community stop misconceiving this brain region are great. How delayed would progress into the nucleus accumbens have been if it were not renamed at some point from its old designation as the nucleus parolfactorious, and likewise, olfacto-striatum?

\section{Going forward}

Major voids exist in our knowledge of the TuS and how it integrates with other neural systems to influence behavioral outcomes. Several matters need to be attended to in order to reach a greater understanding of this neural system. First and foremost, investigators should be mindful of the TuS's unique anatomic features to ensure that studies specifically study the TuS without unintentionally probing or manipulating the immediately adjacent structures, including the ventral pallidum, ventral diagonal band of Broca, or the piriform cortex. A recent study even lumped together the TuS's span as part of the piriform cortex (Carmichael et al., 2017). Thus, careful appreciation of the anatomy of the TuS and especially its borders is needed. Second, investigations into the synaptic circuitry of the TuS are sparse and would aid in resolving input and output from the TuS, as well as how systems within the TuS engage each other to shape information processing. For instance, do D1 and D2 medium spiny neurons in the TuS influence local activity in ways important for learning, as observed recently in the nucleus accumbens? The neurochemical composition of the TuS is rich (Cansler et al., 2020), with a wealth of intrinsic and extrinsic systems in place for investigation. Understanding the microcircuitry of the TuS will be critical for guiding functional studies in behaving animals. Third, studies into the behavioral functions of the TuS will only advance our understanding for this system if the behavior is carefully designed, controlled for, and interpreted. For our understanding of the TuS to be accurately advanced, we should take 
cues from the careful behavioral paradigms used by investigators in other systems (e.g., how Pavlovian fear learning has advanced the understanding of basolateral amygdala, or the vestibular oculomotor reflex has advanced the knowledge of cerebellar cortex) and adapt similar rigorous and sensitive paradigms to the study of the TuS. Finally, and what should be evident by this point, future investigators should not ascribe pure olfactory functions to the TuS, nor for that matter, assign functions associated with one sensory system to most brain regions. I began investigations into the TuS with "olfactory" goggles on; however, our group's work, that of our collaborators, and that of the other groups mentioned here (as well as some that could not be mentioned here for brevity) has shown that the TuS may have a wealth of functions from sensory, cognitive, affective, to motor; and approaching experiments without a bias in what that function may be will be in the best interests of advancing scientific discovery into the roles of this brain region.

\section{References}

Agustín-Pavón C, Martínez-García F, Lanuza E (2014) Focal lesions within the ventral striato-pallidum abolish attraction for male chemosignals in female mice. Behav Brain Res 259:292-296.

Alheid GF, Heimer L (1988) New perspectives in basal forebrain organization of special relevance for neuropsychiatric disorders: the striatopallidal, amygdaloid, and corticopetal components of substantia innominata. Neuroscience 27:1-39.

Anderson AK, Christoff K, Stappen I, Panitz D, Ghahremani DG, Glover G, Gabrieli JD, Sobel N (2003) Dissociated neural representations of intensity and valence in human olfaction. Nat Neurosci 6:196-202.

Cansler HL, Wright KN, Stetzik LA, Wesson DW (2020) Neurochemical organization of the ventral striatum's olfactory tubercle. J Neurochem 152:425-448.

Carelli RM (2002) The nucleus accumbens and reward: neurophysiological investigations in behaving animals. Behav Cogn Neurosci Rev 1:281-296.

Carelli RM, King VC, Hampson RE, Deadwyler SA (1993) Firing patterns of nucleus accumbens neurons during cocaine self-administration in rats. Brain Res 626:14-22.

Carlson KS, Dillione M, Wesson DW (2014) Odor- and state-dependent olfactory tubercle local field potential dynamics in awake rats. J Neurophysiol 111:2109-2123.

Carlson KS, Gadziola MA, Dauster ES, Wesson DW (2018) Selective attention controls olfactory decisions and the neural encoding of odors. Curr Biol 28:2195-2205.e4.

Carmichael JE, Gmaz JM, van der Meer MA (2017) Gamma oscillations in the rat ventral striatum originate in the piriform cortex. J Neurosci 37:7962-7974.

Carriero G, Uva L, Gnatkovsky V, de Curtis M (2009) Distribution of the olfactory fiber input into the olfactory tubercle of the in vitro isolated guinea pig brain. J Neurophysiol 101:1613-1619.

Chiang E, Strowbridge BW (2007) Diversity of neural signals mediated by multiple, burst-firing mechanisms in rat olfactory tubercle neurons. J Neurophysiol 98:2716-2728.

Chikazoe J, Lee DH, Kriegeskorte N, Anderson AK (2014) Population coding of affect across stimuli, modalities and individuals. Nat Neurosci 17:1114-1122.

De Olmos JS, Heimer L (1999) The concepts of the ventral striatopallidal system and extended amygdala. Ann NY Acad Sci 877:1-32.

Desimone R, Duncan J (1995) Neural mechanisms of selective visual. Annu Rev Neurosci 18:193-222.

DiBenedictis BT, Olugbemi AO, Baum MJ, Cherry JA (2014) 6Hydroxydopamine lesions of the anteromedial ventral striatum impair opposite-sex urinary odor preference in female mice. Behav Brain Res 274:243-247.

DiBenedictis BT, Olugbemi AO, Baum MJ, Cherry JA (2015) DREADDinduced silencing of the medial olfactory tubercle disrupts the preference of female mice for opposite-sex chemosignals. eNeuro 2:ENEURO.007815.2015-16.
Fitzgerald BJ, Richardson K, Wesson DW (2014) Olfactory tubercle stimulation alters odor preference behavior and recruits forebrain reward and motivational centers. Front Behav Neurosci 8:81.

Floresco SB (2015) The nucleus accumbens: an interface between cognition, emotion, and action. Annu Rev Psychol 66:25-52.

Fontanini A, Katz DB (2008) Behavioral states, network states, and sensory response variability. J Neurophysiol 100:1160-1168.

Gadziola MA, Wesson DW (2016) The neural representation of goal-directed actions and outcomes in the ventral striatum's olfactory tubercle. J Neurosci 36:548-560.

Gadziola MA, Tylicki KA, Christian DL, Wesson DW (2015) The olfactory tubercle encodes odor valence in behaving mice. J Neurosci 35:45154527.

Gadziola MA, Stetzik LA, Wright KN, Milton AJ, Arakawa K, del Mar Cortijo M, Wesson DW (2020) A neural system for encoding the association of odors with rewarded outcomes and promoted behavioral engagement. Cell Rep 32:107919.

Ganser S (1882) Vergleichend-anatomische Studien uber das Gehrin des Maulwurfs. Morphol Jahrb 7:591-725.

Gottfried JA, O'Doherty J, Dolan RJ (2002) Appetitive and aversive olfactory learning in humans studied using event-related functional magnetic resonance imaging. J Neurosci 22:10829-10837.

Gurdjian ES (1925) Olfactory connections in the albino rat, with special reference to the stria medullaris and the anterior commissure. J Comp Neurol 38:127-163.

Heimer L (1968) Synaptic distribution of centripetal and centrifugal nerve fibres in the olfactory system of the rat: an experimental anatomical study. J Anat 103:413-432.

Heimer L, Switzer RD, Van Hoesen GW (1982) Ventral striatum and ventral pallidum: components of the motor system? Trends Neurosci 5:83-87.

Herrick CJ (1926) Brains of rats and men: a survey of the origin and biological significance of the cerebral cortex. Chicago: University of Chicago.

Hoover WB, Vertes RP (2011) Projections of the medial orbital and ventral orbital cortex in the rat. J Comp Neurol 519:3766-3801.

Howard JD, Kahnt T, Gottfried JA (2016) Converging prefrontal pathways support associative and perceptual features of conditioned stimuli. Nat Commun 7:11546.

Ikemoto S (2003) Involvement of the olfactory tubercle in cocaine reward: intracranial self-administration studies. J Neurosci 23:9305-9311.

Ikemoto S (2007) Dopamine reward circuitry: two projection systems from the ventral midbrain to the nucleus accumbens-olfactory tubercle complex. Brain Res Rev 56:27-78.

Johnson DM, Illig KR, Behan M, Haberly LB (2000) New features of connectivity in piriform cortex visualized by intracellular injection of pyramidal cells suggest that "primary" olfactory cortex functions like "association" cortex in other sensory systems. J Neurosci 20:6974-6982.

Johnston BJ (1913) The morphology of the septum, hippocampus, and pallial commissures in reptiles and mammals. J Comp Neurol 23:371-478.

Johnston BJ (1923) Further contributions to the study of the evolution of the forebrain. J Comp Neurol 35:337-481.

Kalivas PW, Volkow ND (2005) The neural basis of addiction: a pathology of motivation and choice. Am J Psychiatry 162:1403-1413.

Kölliker VA (1896) Handbuch der Gewebelehre des Menschen, Ed 6. Leipzig: Engelmann.

Koob GF, Riley SJ, Smith SC, Robbins TW (1978) Effects of 6-hydroxydopamine lesions of the nucleus accumbens septi and olfactory tubercle on feeding, locomotor activity, and amphetamine anorexia in the rat. J Comp Physiol Psychol 92:917-927.

Kumar S, von Kriegstein K, Friston K, Griffiths TD (2012) Features versus feelings: dissociable representations of the acoustic features and valence of aversive sounds. J Neurosci 32:14184-14192.

Lakatos P, Chen CM, O'Connell MN, Mills A, Schroeder CE (2007) Neuronal oscillations and multisensory interaction in primary auditory cortex. Neuron 53:279-292.

Luskin MB, Price JL (1983a) The laminar distribution of intracortical fibers originating in the olfactory cortex of the rat. J Comp Neurol 216:292302.

Luskin MB, Price JL (1983b) The topographic organization of associational fibers of the olfactory system in the rat, including centrifugal fibers to the olfactory bulb. J Comp Neurol 216:264-291. 
Martin-Lopez E, Xu C, Liberia T, Meller SJ, Greer CA (2019) Embryonic and postnatal development of mouse olfactory tubercle. Mol Cell Neurosci 98:82-96.

McNamara AM, Cleland TA, Linster C (2004) Characterization of the synaptic properties of olfactory bulb projections. Chem Senses 29:225-233.

Meyer G, Wahle P (1986) The olfactory tubercle of the cat: I. Morphological components. Exp Brain Res 62:515-527.

Millhouse OE (1987) Granule cells of the olfactory tubercle and the question of the islands of Calleja. J Comp Neurol 265:1-24.

Millhouse OE, Heimer L (1984) Cell configurations in the olfactory tubercle of the rat. J Comp Neurol 228:571-597.

Millman DJ, Murthy VN (2020) Rapid learning of odor-value association in the olfactory striatum. J Neurosci 40:4335-4347.

Mingote S, Chuhma N, Kusnoor SV, Field B, Deutch AY, Rayport S (2015) Functional connectome analysis of dopamine neuron glutamatergic connections in forebrain regions. J Neurosci 35:16259-16271.

Murata K, Kanno M, Ieki N, Mori K, Yamaguchi M (2015) Mapping of learned odor-induced motivated behaviors in the mouse olfactory tubercle. J Neurosci 35:10581-10599.

Nicola SM (2007) The nucleus accumbens as part of a basal ganglia action selection circuit. Psychopharmacology (Berl) 191:521-550.

O'Connell LA, Hofmann HA (2011) The vertebrate mesolimbic reward system and social behavior network: a comparative synthesis. J Comp Neurol 519:3599-3639.

Oettl LL, Scheller M, Filosa C, Wieland S, Haag F, Loeb C, Durstewitz D, Shusterman R, Russo E, Kelsch W (2020) Phasic dopamine reinforces distinct striatal stimulus encoding in the olfactory tubercle driving dopaminergic reward prediction. Nat Commun 11:3460.

Paxinos G, Franklin K (2000) The mouse brain in stereotaxic coordinates, Ed 2. San Diego: Academic.

Payton CA, Wilson DA, Wesson DW (2012) Parallel odor processing by two anatomically distinct olfactory bulb target structures. PLoS One 7:e34926.

Pigache RM (1970) The anatomy of "paleocortex": a critical review. Ergeb Anat Entwicklungsgesch 43:3-62.

Prado-Alcalá R, Wise RA (1984) Brain stimulation reward and dopamine terminal fields: I. Caudate-putamen, nucleus accumbens and amygdala. Brain Res 297:265-273.

Ramon y Cajal S (1904) Textura del sistema nervioso del hombre y de los vertebrados. Madrid: Impr Libr Nicolás Moya.

Russo SJ, Nestler EJ (2013) The brain reward circuitry in mood disorders. Nat Rev Neurosci 14:609-625.

Schwob JE, Price JL (1984a) The development of lamination of afferent fibers to the olfactory cortex in rats, with additional observations in the adult. J Comp Neurol 223:203-222.

Schwob JE, Price JL (1984b) The development of axonal connections in the central olfactory system of rats. J Comp Neurol 223:177-202.

Scott JW (1981) Electrophysiological identification of mitral and tufted cells and distributions of their axons in olfactory system of the rat. J Neurophysiol 46:918-931.

Scott JW, McBride RL, Schneider SP (1980) The organization of projections from the olfactory bulb to the piriform cortex and olfactory tubercle in the rat. J Comp Neurol 194:519-534.
Simpson D (2005) Phrenology and the neurosciences: contributions of F. J. Gall and J. G. Spurzheim. ANZ J Surg 75:475-482.

Sosulski DL, Lissitsyna Bloom M, Cutforth T, Axel R, Datta SR (2011) Distinct representations of olfactory information in different cortical centres. Nature 472:213-216.

Striano BM, Barker DJ, Pawlak AP, Root DH, Fabbricatore AT, Coffey KC, Stamos JP, West MO (2014) Olfactory tubercle neurons exhibit slow-phasic firing patterns during cocaine self-administration. Synapse 68:321323.

Switzer RC, Hill J, Heimer L (1982) The globus pallidus and its rostroventral extension into the olfactory tubercle of the rat: a cyto- and chemoarchitectural study. Neuroscience 7:1891-1904.

Vertes RP (2004) Differential projections of the infralimbic and prelimbic cortex in the rat. Synapse 51:32-58.

Wesson DW, Wilson DA (2010) Smelling sounds: olfactory-auditory sensory convergence in the olfactory tubercle. J Neurosci 30:3013-3021.

Wesson DW, Wilson DA (2011) Sniffing out the contributions of the olfactory tubercle to the sense of smell: hedonics, sensory integration, and more? Neurosci Biobehav Rev 35:655-668.

West EA, Carelli RM (2016) Nucleus accumbens core and shell differentially encode reward-associated cues after reinforcer devaluation. J Neurosci 36:1128-1139.

White KA, Zhang YF, Zhang Z, Bhattarai JP, Moberly AH, In 't Zandt EE, Peña-Bravo JI, Mi H, Jia X, Fuccillo MV, Xu F, Ma M, Wesson DW (2019) Glutamatergic neurons in the piriform cortex influence the activity of D1 and D2-type receptor expressing olfactory tubercle neurons. J Neurosci 39:9546-9559.

White LE (1965) Olfactory bulb projections of the rat. Anat Rec 152:465479.

Wieland S, Schindler S, Huber C, Köhr G, Oswald MJ, Kelsch W (2015) Phasic dopamine modifies sensory-driven output of striatal neurons through synaptic plasticity. J Neurosci 35:9946-9956.

Williams GV, Rolls ET, Leonard CM, Stern C (1993) Neuronal responses in the ventral striatum of the behaving macaque. Behav Brain Res 55:243252.

Xia CZ, Adjei S, Wesson DW (2015) Coding of odor stimulus features among secondary olfactory structures. J Neurophysiol 114:736-745.

Zald DH (2003) The human amygdala and the emotional evaluation of sensory stimuli. Brain Res Brain Res Rev 41:88-123.

Zelano C, Bensafi M, Porter J, Mainland J, Johnson B, Bremner E, Telles C, Khan R, Sobel N (2005) Attentional modulation in human primary olfactory cortex. Nat Neurosci 8:114-120.

Zhang Z, Liu Q, Wen P, Zhang J, Rao X, Zhou Z, Zhang H, He X, Li J, Zhou Z, Xu X, Zhang X, Luo R, Lv G, Li H, Cao P, Wang L, Xu F (2017a) Activation of the dopaminergic pathway from VTA to the medial olfactory tubercle generates odor-preference and reward. Elife 6:e25423.

Zhang Z, Zhang H, Wen P, Zhu X, Wang L, Liu Q, Wang J, He X, Wang H, $\mathrm{Xu} \mathrm{F}$ (2017b) Whole-brain mapping of the inputs and outputs of the medial part of the olfactory tubercle. Front Neurol Circuits 11:52. 